\title{
Caracterización aromática de variedades minoritarias del Piedemonte Pirenaico
}

\author{
Ricardo López ${ }^{1 *}$, Laura Culleré ${ }^{1}$,Elisa Gracia-Moreno ${ }^{1},{\text { Fanny } \text { Prezman }^{2} \text { y Ernesto Franco Aladrén }}^{3}$ \\ ${ }^{1}$ Laboratorio de Análisis del Aroma y Enología, Instituto Agroalimentario de Aragón (IA2), Departamento de Química Analítica, \\ Facultad de Ciencias, Universidad de Zaragoza, E-50009 Zaragoza, España \\ ${ }^{2}$ Institut de la Vigne et du Vin- Pôle Sud Ouest. Lisle Sur Tarn. Francia \\ ${ }^{3}$ Unidad de Enología. Centro de Transferencia Agroalimentaria. Zaragoza. España
}

\begin{abstract}
Resumen. Mediante los conservatorios de variedades y la colaboración ciudadana se han identificado más de un centenar de variedades minoritarias procedentes de las regiones que rodean los Pirineos. Con 78 de dichas variedades identificadas se han realizado más de 200 microvinificaciones que han permitido, por una parte, evaluar su potencial enológico y, por otra, caracterizar sensorial y químicamente dichas variedades.
\end{abstract}

\section{Introducción}

La globalización del mercado del vino durante los últimos treinta años ha provocado una disminución de las variedades de uva utilizadas para vinificación. En la actualidad de las más de 10000 variedades de uva conocidas, 13 cubren más de un tercio de la superficie plantada y 33 variedades cubren el 50\% [1]. En España esta situación es también extrema, ya que sólo 10 variedades representan el $75 \%$ de la superficie plantada.

Este fenómeno de homogenización de las variedades empleadas para vinificación conlleva un empobrecimiento genético a causa de la selección clonal y una dificultad de los pequeños productores para conseguir competitividad en un mercado cada vez más global.

Una posible solución a estos problemas puede encontrarse en las variedades de vid minoritarias empleadas como herramienta de innovación. Las regiones francesas y españolas que rodean a los Pirineos han tenido una gran diversidad de variedades de vid. Esto es debido a su larga tradición como región vitícola, ya desde tiempos del Imperio Romano, pasando por los intercambios culturales del Camino de Santiago y, por supuesto, por su gran variedad geográfica. Gracias a prospecciones llevadas a cabo estos 10 últimos años en antiguas parcelas vitícolas, se han implantado diversos conservatorios o colecciones varietales en estas regiones. Sin embargo, el potencial agronómico y enológico de dichas variedades minoritarias aún está pendiente de ser evaluado.

Este es el objetivo del proyecto europeo "Valorización de las variedades de vid minoritarias del Piedemonte Pirenáico" (VALOVITIS). Dentro de este proyecto, se ha identificado de manera exhaustiva más de un centenar de variedades procedentes de las regiones que rodean los Pirineos. Con las variedades identificadas se han realizado más de 200 microvinificaciones. Los resultados de la caracterización de las diferencias varietales en lo que respecta a su composición en compuestos químicos volátiles se presenta en esta comunicación.

\section{Material y métodos}

\subsection{Variedades}

Un total de 78 variedades minoritarias se emplearon para elaborar los vinos. Las variedades utilizadas procedían del conservatorio de variedades del Centro de Transferencia Agroalimentaria del Gobierno de Aragón del conservatorio de variedades del Institut de la Vigne et du Vin- Pôle Sud Ouest en Francia.

\subsection{Vinificaciones}

Las fermentaciones y tratamientos de las variedades españolas se realizaron en la bodega experimental del Gobierno de Aragón en Cariñena, mientras que las de las variedades francesas se realizaron en las instalaciones del Institut de la Vigne et du Vin- Pôle Sud Ouest en Francia. Debido al escaso número de plantas disponibles para cada variedad los vinos se elaboraron a escala de microvinificación $(5 \mathrm{~L})$, siguiendo un protocolo estándar común para las vinificaciones en tinto, rosado y blanco en cada país. Las vinificaciones se realizaron con las cosechas de 2016 y 2017.

\subsection{Análisis cuantitativo de compuestos volátiles}

Un total de 82 compuestos volátiles implicados en el aroma del vino se analizaron en todas las muestras, Para ello se utilizaron cuatro métodos de análisis diferentes,

Corresponding author: riclopez@unizar.es 
todos ellos publicados y validados. Los compuestos mayoritarios (aquellos con concentraciones en el rango de $\mathrm{mg} / \mathrm{l}$ ) se analizaron mediante microextracción líquidolíquido y cromatografía gas (GC) con detección FID [2]. Los compuestos minoritarios y traza (concentraciones por debajo de $1 \mathrm{mg} / \mathrm{l}$ hasta el $\mu \mathrm{g} / \mathrm{l}$ ) se analizaron mediante extracción en fase sólida (SPE) y GC con detección por espectrometría de masas (MS) [3]. Los tioles varietales (en concentraciones de $\mathrm{ng} / \mathrm{L}$ ) se determinaron mediante SPE y GC-MS con ionización química negativa [4]. Por último, las alquilmetoxipirazinas y rotundona se analizaron mediante SBSE y cromatografía gas multidimensional GC-GC-MS [5].

\subsection{Análisis estadístico}

Los análisis estadísticos se llevaron a cabo con el programa XLSTAT 19.6 (Addinsoft).

\section{Resultados y discusión}

Es de sobras conocido que existe una parte del aroma del vino cuyo origen está en gran parte determinado por la variedad de uva utilizada, es lo que se conoce como aroma varietal. A la hora de evaluar el potencial aromático de las variedades minoritarias, parece lógico analizar su capacidad de producción de las moléculas volátiles responsables de estos aromas varietales. Por esta razón se ha dividido el estudio de los resultados de composición química de acuerdo con las familias de compuestos varietales más interesantes. A continuación, se muestra un resumen de los hallazgos más relevantes.

\subsection{Tioles varietales}

Existen varios mercaptanos polifuncionales que pueden ser responsables de aromas positivos y cuya presencia en el vino está asociada a la variedad de uva utilizada. Los más destacados en este grupo son la 4-metil-4-mercapto2-pentanona identificada como un compuesto clave en el aroma del Sauvignon blanc [6], el 3-mercaptohexanol y el acetato de mercaptohexilo también compuestos impacto en los vinos de Verdejo y de Sauvignon blanc entre otros [4]. Entre las variedades minoritarias estudiadas hay varias que destacan por su contenido en tioles varietales. En la figura 1 puede observarse como los vinos de 6 variedades minoritarias mostraron contenidos superiores a 10 veces el umbral sensorial del 3-mercaptohexanol. Estos niveles son comparables a los encontrados en los vinos de Sauvignon blanc y producen en el vino notas de fruta tropical [4]. El vino rosado elaborado con la variedad San Antoni también destacó por un nivel muy alto en acetato de mercaptohexilo.

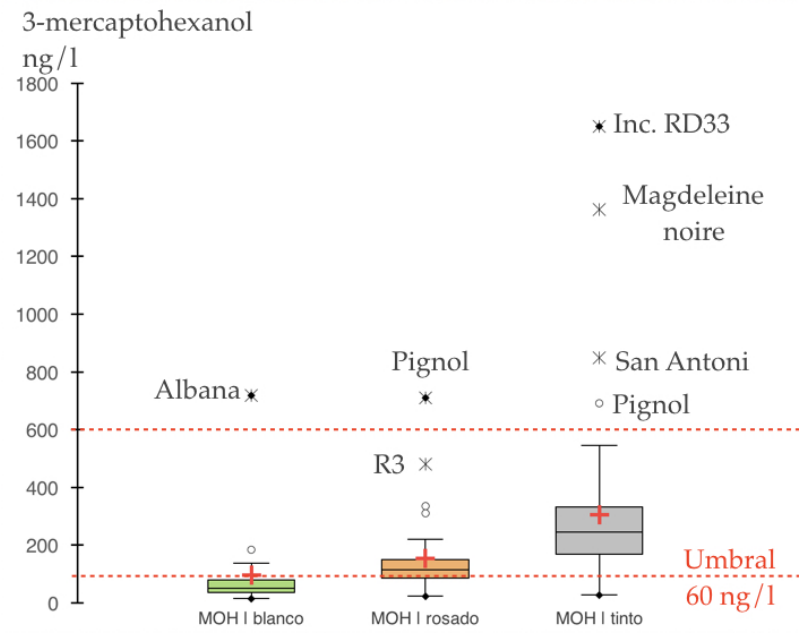

Fig. 1. Diagrama de cajas para el contenido en 3mercaptohexanol en los vinos de variedades minoritarias

\subsection{Rotundona}

La rotundona es un sesquiterpeno de origen varietal y gran potencia sensorial, ya que a partir de los $16 \mathrm{ng} / \mathrm{L}$ imparte una característica y apreciada nota de pimienta negra [7]. La rotundona ha sido localizada en otras variedades originarias de la zona Pirenaica como el Duras [8]. Entre los vinos producidos en este trabajo hubo 7 variedades que destacaron por su contenido en rotundona, pero dos de ellas (Negral y Camarou) mostraron niveles claramente por encima del valor umbral (Figura 2). Estas mismas variedades elaboradas como vino rosado no mostraron niveles apreciables de rotundona, debido a que su precursor sólo se encuentra en los hollejos.

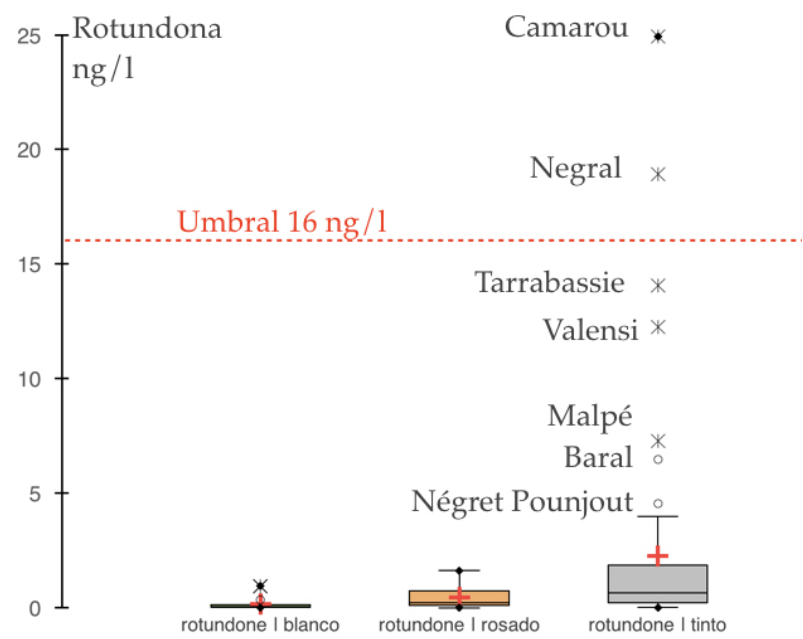

Fig. 2. Diagrama de cajas para el contenido en rotundona en los vinos de variedades minoritarias

\subsection{Terpenoles y norisoprenoides}

Los vinos producidos no destacaron por niveles fuera de lo normal de norisoprenoides con relevancia para el 
aroma como $\beta$-damascenona o $\beta$-ionona. Sin embargo, es destacable que los vinos españoles contenían un nivel medio mayor de estos compuestos, debido probablemente al mayor número de horas de insolación. No se encontraron niveles significativos de TDN. Respecto a los terpenoles, sólo una de las variedades mostró niveles por encima del valor umbral de linalool en la elaboración en rosado, confirmando su carácter terpenodependiente.

\subsection{Alquilmetoxipirazinas}

Sólo dos de las variedades produjeron vinos con niveles superiores o cercanos al valor umbral sensorial de 3isobutil-2-metoxipirazina. En ambos casos se trataba de variedades francesas: Gros Cabernet con $12 \mathrm{ng} / \mathrm{L}$ y Bequignol con $42 \mathrm{ng} / \mathrm{L}$. Especialmente en el último caso aparecieron notas vegetales debido a este compuesto.

\subsection{Compuestos fermentativos}

Aunque la producción de compuestos fermentativos está fundamentalmente controlada por la levadura utilizada, existe también una influencia varietal a través del perfil de aminoácidos característico de la variedad [9]. En el caso de las variedades minoritarias estudiadas se encontraron diferencias significativas en algunos compuestos fermentativos importantes desde el punto de vista del aroma. Entre otros destacaron acetato de isoamilo, acetato de feniletilo o $\gamma$-nonalactona. En la Figura 3 puede observarse el caso del acetato de isoamilo, que en la variedad Plant de Fronton mostró una cantidad muy llamativa, con una cantidad cercana a los 8 $\mathrm{mg} / \mathrm{L}$.

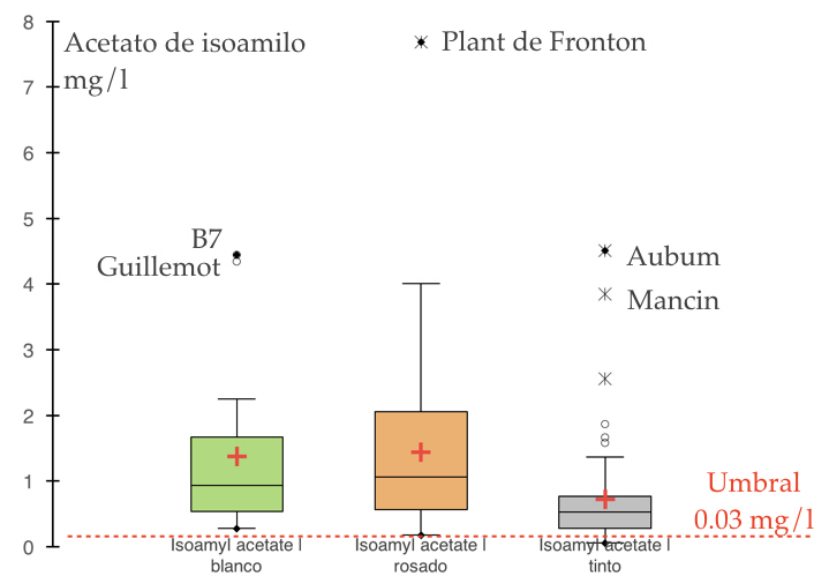

Fig. 2. Diagrama de cajas para el contenido de acetato de isoamilo en los vinos de variedades minoritarias

\section{Conclusiones}

Muchas de las variedades minoritarias localizadas presentan un potencial enológico, desde el punto de vista sensorial, altamente interesante. Algunos de los vinos elaborados contienen compuestos de gran impacto sensorial en cantidades similares a las de variedades muy apreciadas como Sauvignon blanc o Shiraz. El trabajo realizado dentro de este proyecto abre la puerta a una futura implantación de estas variedades en las regiones pirenaicas como una herramienta de mejora de la calidad y la competitividad.

VALOVITIS es un proyecto de colaboración transfronteriza entre España y Francia cofinanciado por el Fondo Europeo de Desarrollo Regional (FEDER) a través del Programa Interreg V-A España-Francia-Andorra (POCTEFA 2014-2020), destinado a reforzar la integración económica y social de la zona fronteriza España-Francia-Andorra.

\section{Referencias}

[1] Distribution of the world's grapevine varieties, International Organisation of Vine and Wine (OIV), 2017.

[2] C. Ortega, R. López, J. Cacho, V. Ferreira, J. Chromatogr. A., 923 (2001).

[3] R. Lopez, M. Aznar, J. Cacho, V. Ferreira, J. Chromatogr. A., 966 (2002).

[4] L. Mateo-Vivaracho, J. Zapata, J. Cacho, V. Ferreira, J. Agric. Food Chem., 58 (2010).

[5] Y. Wen, I. Ontañón, V. Ferreira, R. Lopez, Food Chem., 255 (2018).

[6] P. Darriet, T. Tominaga, V. Lavigne, J.N. Boidron, D. Dubourdieu, Flavour Fragrance J., 10 (1995).

[7] C. Wood, T.E. Siebert, M. Parker, D.L. Capone, G.M. Elsey, A.P. Pollnitz, et al., J. Agric. Food Chem., 56 (2008).

[8] O. Geffroy, T. Dufourcq, D. Carcenac, T. Siebert, M. Herderich, E. Serrano, Ais. J. Grape Wine Res., 3 (2014).

[9] P. Hernandez-Orte, J.F. Cacho, V. Ferreira, J. Agric. Food Chem. 50 (2002). 